\title{
Pacientes amputados por accidentes de trabajo: características y años acumulados de vida productiva potencial perdidos
}

\author{
Patients amputated by work accidents: characteristics and years accumulated of potential \\ productive life lost
}

\author{
Herminio Teófilo Camacho-Conchucos ${ }^{1}$ \\ ${ }^{1}$ Instituto Nacional de Rehabilitación.
}

\begin{abstract}
Resumen
Objetivos: Determinar los años acumulados de vida productiva potencial perdidos en pacientes amputados por accidentes de trabajo. Diseño: Estudio descriptivo, retrospectivo, de corte transversal. Institución: Instituto Nacional de Rehabilitación, Callao, Perú. Participantes: Pacientes amputados por accidente de trabajo. Intervenciones: Revisión de 1290 historias clínicas de pacientes amputados, atendidos del 1 de enero del 2003 al 31 de diciembre del 2007, de los cuales 108 fueron por accidente de trabajo. Se determinó los años acumulados de vida productiva potencial perdidos, multiplicando la diferencia entre la edad de retiro y la edad al momento de dictaminarse el grado de invalidez permanente, por el porcentaje de la invalidez. Principales medidas de resultados: Años acumulados de vida productiva potencial perdidos. Resultados: El accidente de trabajo fue la causa en 8,4\% de los amputados, que generaron 1 568,5 años acumulados de vida productiva potencial perdidos, con 14,5 años por cada caso. El nivel más frecuente fue debajo de rodilla, con $27,3 \%$. La manufactura fue la actividad económica más frecuente, con 37,9\%, y la forma de accidente fue el aprisionamiento o atrapamiento en $58,3 \%$. El sexo masculino predominó, 98,2\%. El $40,7 \%$ se encontró en el rango de 21 a 30 años de edad y $63,9 \%$ no realizará su labor habitual por su invalidez. Conclusiones: Los años acumulados de vida productiva potencial perdidos es un indicador de gran utilidad para complementar la evaluación de los accidentes de trabajo en la invalidez residual, porque considera la edad en que se produce la invalidez y el porcentaje de la misma.

Palabras clave: Amputación; accidentes de trabajo; incapacitados.
\end{abstract}

\begin{abstract}
Objectives: To determine the accumulated years of potential productive life lost in amputated patients by work accidents. Design: Descriptive, retrospective, cross-sectional study. Setting: Instituto Nacional de Rehabilitacion, Callao, Peru. Participants: Amputated patients by work accidents. Interventions: Review of 1290 medical records of amputated patients attended from January 1, 2003, to December 31, 2007, 108 due to work accidents. The accumulated years of potential productive life lost were determined multiplying the difference between the age of retirement and the age at the time of considering the degree of permanent disability by the percentage of the disability. Main outcome results: Accumulated years of potential productive life lost. Results: Work accident caused $8,4 \%$ of amputations generating 1568,5 accumulated years of potential productive life lost, with 14,5 years in each case. The most frequent site of amputation was below the knee in $27,3 \%$. Manufacture was the most frequent economic activity in $37,9 \%$ and the accident was imprisonment or entrapment in $58,3 \%$. Male sex predominated in $98,2 \% ; 40,7 \%$ were in the range 21 to 30 years of age and $63,9 \%$ will not be able to make their habitual work due to disability. Conclusions: The accumulated years of potential productive life lost is a very useful indicator complementing evaluation of work accidents in residual disability as it considers age at disability and its percentage.

Keywords: Amputation; accidents, occupational; disabled.
\end{abstract}

\section{INTRODUCCIÓN}

El trabajo es una actividad que dignifica al hombre, satisface sus necesidades y permite su desarrollo. Se conoce que cuando el trabajo se desarrolla en condiciones que pueden causar daño a la integridad física y a su salud, se producen accidentes y enfermedades ${ }^{(1)}$.

El accidente de trabajo es definido como todo suceso repentino y prevenible que sobrevenga por causa o con ocasión del trabajo, y que produzca en el trabajador una lesión orgánica, una perturbación funcional, una invalidez o muerte. Es también accidente de trabajo aquel que se produce durante la ejecución de órdenes del empleador, o durante la ejecución de una labor bajo su autoridad, aún fuera del lugar de trabajo ${ }^{(2)}$.

Los accidentes de trabajo son la combinación de riesgo físico y error humano y causan lesión de la persona ${ }^{(3)}$. Ocurrido el accidente, puede generar un cambio en la vida de la persona al ocasionar una incapacidad temporal, en el mejor de los casos, es decir una incapacidad para trabajar por un tiempo. Lo peor es cuando queda con una secuela permanente que lo limita de por vida para su labor habitual o para cualquier actividad laboral, quedando con una incapacidad o invalidez, término utilizado para el pago de pensiones ${ }^{(1,4,5)}$. La amputación constituye uno de los daños más graves, después de la muerte en un accidente de trabajo. Ocasiona una incapacidad permanente en el trabajador, para su ocupación y para su vida misma. Genera un shock psicológico, por la pérdida de un miembro, que lo hace diferente a las demás personas, por ser una diferencia evidente $^{(6,7)}$.

El diagnóstico situacional de los accidentes de trabajo en el país es dificultado por la falta de registro o el subregistro existente en las instituciones comprometidas con la vigilancia de accidentes de trabajo. En el 2004, EsSalud comunicó 13693 casos de accidentes de trabajo, existiendo un subregistro de $46 \%$, es decir, el sector formal estaría bordeando los 19000 casos. Si a esta cifra sumamos el 60\% representado por el sector infor- 
mal, la cifra llegaría a 50000 casos, o 135 casos diarios $^{(8)}$.

El estudio de accidentes implica la aplicación de indicadores para determinar las causas y las consecuencias para mejorar las acciones preventivas. Los más utilizados son: índice de frecuencia, que expresa cuantos accidentes ocurren por cada millón de horas trabajadas; índice de gravedad, que representa el número de jornadas perdidas por cada mil horas de exposición al riesgo (las trabajadas) ${ }^{(9)}$; años de vida ajustados a discapacidad, que es la medición simultánea de las consecuencias mortales y no mortales de las intervenciones sanitarias, lo que ha dado origen a la cuantificación de la carga de enfermedad ${ }^{(10)}$; años de vida productiva potencialmente perdidos (AVPPP), que son los años de vida productivos potenciales perdidos para el país, a causa de mortalidad prematura; años de vida potenciales perdidos (AVPP), que son los años de vida potenciales que se pierden para el país a causa de la mortalidad prematura ${ }^{(2)}$.

La investigación sobre factores determinantes del impacto económico de los años de vida productiva potencialmente perdidos (AVPPP) y la magnitud de los años de vida potencialmente perdidos (AVPP) ocasionados por accidentes de trabajo mortales en la minería peruana, 1994-1999, encontró en 491 trabajadores 15 407,12 años de vida potenciales perdidos y 5891 años de vida productiva potencialmente perdidos ${ }^{(11)}$. En España, un estudio sobre estimación de la mortalidad atribuible a enfermedades laborales en España, 2004, encontró cerca de 16000 muertes por enfermedades relacionadas con exposiciones laborales. Estas muertes habrían causado cerca de 152000 años de vida potencialmente perdidos y algo más de 47000 años de vida productiva potencialmente perdidos ${ }^{(12)}$.

Sin embargo, estos indicadores, que son los más utilizados, reflejan principalmente el costo económico y humano subvaluado, sin tomar en cuenta a la vez la incapacidad residual, el momento de la vida productiva en que ocurre la lesión y la correspondiente disminución en la capacidad productiva del trabajador.
Años acumulados de vida productiva potencial perdidos (AAVPPP) es un indicador que, a diferencia de los anteriores, no utiliza datos de mortalidad o estimaciones promedio de invalidez posterior a la enfermedad, sino que aplica porcentajes de incapacidad permanente individual. La incapacidad permanente se obtiene como invalidez en base a tablas de menoscabo, derivado de los impedimentos físicos o mentales y la asociación de los factores complementarios ${ }^{(4)}$. El indicador en mención, se aproxima al daño por accidente de trabajo con una mayor objetividad y permitiría una compensación más justa, porque toma en cuenta la edad en que se produce la invalidez, el grado de la misma expresada en porcentaje y la edad de jubilación.

En un estudio en México, se aplicó el indicador de AAVPPP en accidentes de trabajo en petróleos mexicanos, con el propósito de complementar la evaluación de las pérdidas por accidentes de trabajo. De 1685 accidentes de trabajo que quedaron con algún grado de secuela, se encontró 5611 AAVPPP, con un promedio por caso de 3,32 años ${ }^{(13)}$.

Por tanto, el presente estudio tuvo como objetivo determinar los años acumulados de vida productiva potencial perdidos en pacientes amputados por accidentes de trabajo, por ser un indicador que valora los años de producción que pierden los trabajadores accidentados en ejercicio o con motivo de su labor, producto del grado de invalidez que presentan.

\section{MÉTODOS}

Se realizó un estudio descriptivo, retrospectivo, de corte transversal. La población la constituyeron todos los pacientes amputados por accidente laboral, atendidos en el Servicio de Amputados y Quemados del Instituto Nacional de Rehabilitación ${ }^{(14)}$ del 1 de enero de 2003 al 31 de diciembre de 2007.

De las historias clínicas de los pacientes seleccionados se obtuvo el nivel de amputación y datos generales. Así mismo, se utilizó el certificado de discapacidad como base para la calificación de invalidez, en los casos en que no había sido determinado, utilizando las tablas de menoscabo para amputados del Manual de Evaluación y Calificación del Grado de Invalidez; se obtuvo en base al nivel de amputación un porcentaje de menoscabo global. En aquellos casos en que se encontraban afectados dos o más órganos o sistemas, los valores parciales de los menoscabos respectivos deben ser combinados, según la fórmula $\mathrm{A} \%$ $+\mathrm{B} \% \times(100 \%-\mathrm{A} \%)$, que combina los valores correspondientes de A y B. Este procedimiento se facilita utilizando la tabla de valores combinados. Finalmente, se asocia los factores complementarios, que son asignaciones porcentuales de menoscabo que se agregan al grado de invalidez, por concepto de edad, grado de instrucción y desempeño de labor habitual, permitiendo determinar el grado y tipo de invalidez ${ }^{(4)}$.

Contando con el porcentaje de invalidez, se determina los años acumulados de vida productiva potencial perdidos mediante la siguiente fórmula:

$$
\text { AAVPPP = (ES-EA) }(\mathrm{I} / 100)
$$

Donde:

AAVPPP = Años acumulados de vida productiva potencial perdidos.

$\mathrm{ES}=$ Edad estándar de retiro $(65$ años) ${ }^{(15)}$.

$\mathrm{EA}=$ Edad al momento de dictaminarse el grado de incapacidad permanente

I = Incapacidad o invalidez permanente en porcentaje.

Se elaboró una base de datos con el programa SPSS 12. Se efectuó el cálculo de los años acumulados de vida productiva potencial perdidos individualmente y para el total de la población. Con estos valores se hizo el cruce con las otras variables establecidas para el estudio, de manera de presentar las tablas respectivas.

El análisis estadístico se realizó sobre la base de parámetros descriptivos generales, de acuerdo a los objetivos planteados. Se determinó promedios, desviación estándar y coeficiente de variación, para medir el grado de dispersión de los datos. 
Tabla 1. Años acumulados de vida productiva potencial perdidos según nivel de amputación por accidente laboral.

\begin{tabular}{lrrr}
\hline Nivel de amputación & $\mathrm{N}^{\circ}$ & $\%$ & AAVPPP \\
\hline Debajo de rodilla & 30 & 27,8 & 383,1 \\
Dedos de la mano & 24 & 22,2 & 165,5 \\
Arriba de rodilla & 14 & 12,9 & 224,5 \\
Debajo del codo & 12 & 11,1 & 259,7 \\
Arriba del codo & 8 & 7,4 & 177,0 \\
Combinado & 6 & 5,6 & 158,5 \\
Tarso & 3 & 2,8 & 29,9 \\
Transmetacarpiano & 2 & 1,9 & 48,4 \\
Metatarsofalángica & 2 & 1,9 & 15,9 \\
Metacarpo & 2 & 1,9 & 22,7 \\
Desarticulado de codo & 1 & 0,9 & 20,3 \\
Hemipelvectomía & 1 & 0,9 & 27,5 \\
Desarticulado de hombro & 1 & 0,9 & 4,3 \\
Desarticulado de cadera & 1 & 0,9 & 18,5 \\
Dedos del pie & 1 & 0,9 & 12,5 \\
Total & 108 & 100 & 1568,5 \\
\hline
\end{tabular}

AAVPPP: Años acumulados de vida productiva potencial perdidos.

\section{RESULTADOS}

Durante el periodo de estudio se atendió 1290 pacientes amputados. La etiología traumática ocupó el primer lugar, con 764 (59,2\%), vascular 247 (19,2\%), neoplasia $111(8,6 \%)$, congénita $86(6,7 \%)$, infecciones $46(3,6 \%)$ y otras $36(2,8 \%)$. Del total de esta población de pacientes amputados, 108 (8,4\%) fueron por accidente de trabajo.

En la distribución de los pacientes amputados por año, se encontró en el 2003 un total de 396 pacientes amputados; 34 fueron por accidente de trabajo $(8,6 \%)$; el 2004, de 250, 35 (14\%) fueron por accidente de trabajo; el 2005, de 208, 16 (7,7\%); el 2006, de 211, 10 (4,7\%); y el 2007 , de $225,13(5,8 \%)$, por la misma causa.

Las edades de los 108 pacientes en el momento de la amputación por accidente de trabajo fueron de 11 a 63 años. El rango de edad predominante fue de 21 a 30 años, con $40,7 \%$, seguido por el de 31 a 40 años, con $19,4 \%$. La mayor cantidad de pacientes amputados por accidente laboral se encontró en los rangos de 21 a 50 años, con $78,7 \%$. En relación al sexo, 106 (98,2\%) fueron masculinos y $2(1,9 \%)$ del sexo femenino.
Los pacientes amputados por accidente de trabajo generaron, de acuerdo a la aplicación del indicador para el estudio, 1568,5 años acumulados de vida productiva potencial perdidos AAVPP, con 14,5 AAVPP como promedio.

El nivel de amputación más frecuente por accidente de trabajo fue debajo de la rodilla con $27,3 \%$, seguido por el de dedos de la mano, con 22,2\%. El nivel con mayor cantidad de años acumulados de vida productiva potencial perdidos fue también el de debajo de rodilla, con 383,1 , pero seguido por el debajo de codo con 259,65 AAVPPP (tabla 1).

La distribución de los 1 568,5 años acumulados de vida productiva potencial perdidos en los 5 años de estudio, tuvo una tendencia decreciente. En el 2003, fue 568,0 AAVPPP y, en el 2007, solo llegó a 153,1 AAVPPP. El promedio por cada caso igualmente fue alto en el primer año, con 16,7 AAVPPP, disminuyendo en los siguientes años, con un ligero aumento en el año 2006, con 15,9 AAVPPP (figura 1).

La actividad económica con mayor número de casos fue la manufactura, con $37,9 \%$, seguido de transporte, almacenamiento y comunicaciones, con $22,2 \%$. También, en los años acumulados de vida productiva potencial perdidos fue mayor en manufactura, pero el mayor promedio por caso correspondió a la administración pública y defensa, seguido de explotación de minas y canteras (tabla 2).

La forma de accidente más frecuente fue el aprisionamiento o atrapamiento, en $58,3 \%$, seguido por el de choque de vehículos, con $12 \%$. El rango de invalidez que presentó la mayoría de los pacientes

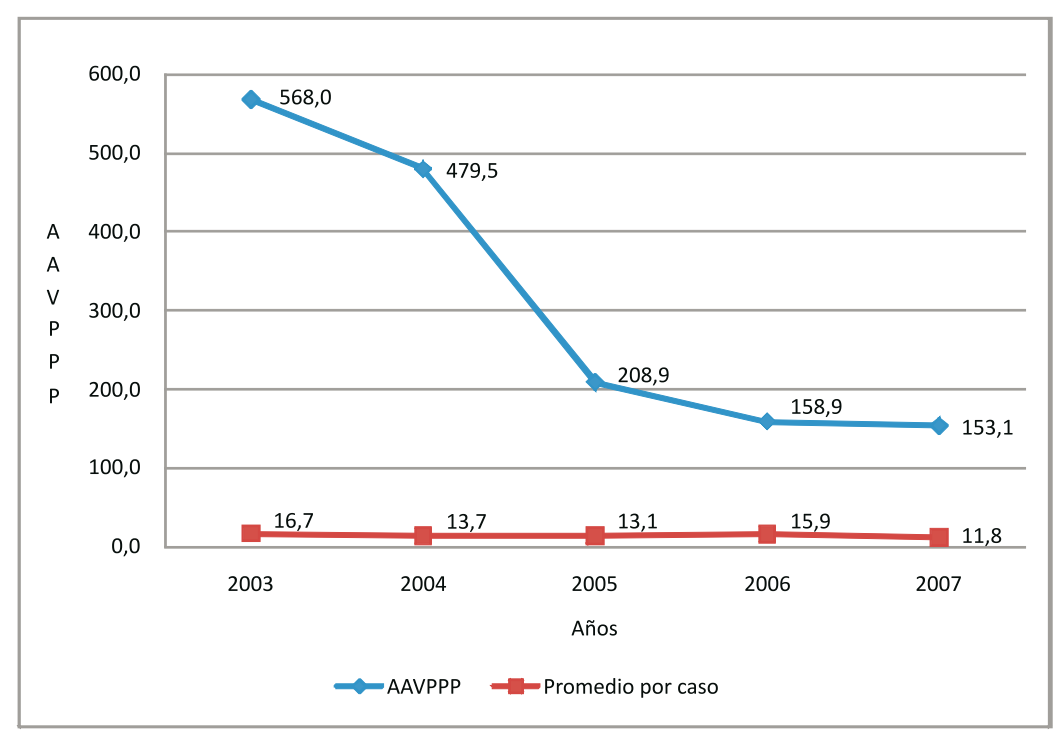

Figura 1. Años acumulados de vida productiva potencial perdidos y promedio por caso, en pacientes amputados por accidente laboral. 
Tabla 2. Años acumulados de vida productiva potencial perdidos según actividad económica en pacientes amputados por accidente laboral.

\begin{tabular}{|c|c|c|c|c|c|c|}
\hline Actividad económica & $\mathrm{N}^{0}$ & $\%$ & AAVPPP & AAVPPP por caso & DE & C V \\
\hline Industrias manufactureras & 41 & 37,9 & 577,0 & 14,1 & 7,8 & 55,73 \\
\hline Transporte, almacenamiento y comunicaciones & 24 & 22,2 & 347,2 & 14,5 & 8,1 & 56,28 \\
\hline Agricultura, ganadería, caza y silvicultura & 10 & 9,3 & 124,3 & 12,4 & 9,8 & 78,61 \\
\hline Administración pública y defensa & 6 & 5,6 & 114,4 & 19,1 & 10,3 & 53,74 \\
\hline Construcción & 8 & 7,4 & 104,3 & 13,0 & 12,1 & 92,55 \\
\hline Explotación de minas y canteras & 5 & 4,6 & 94,8 & 18,9 & 6,1 & 32,16 \\
\hline Pesca & 6 & 5,6 & 79,7 & 13,3 & 8,3 & 62,76 \\
\hline Suministro de electricidad, gas y agua & 4 & 3,7 & 60,8 & 15,2 & 8,1 & 53,33 \\
\hline Otras actividades de servicios comunitarios, sociales y personales & 2 & 1,9 & 34,5 & 17,3 & 4,6 & 26,84 \\
\hline Comercio al por mayor y al por menor & 2 & 1,9 & 31,6 & 15,8 & 17,5 & 110,81 \\
\hline Total & 108 & 100 & 1568,5 & 14,5 & 8,5 & 58,53 \\
\hline
\end{tabular}

AAVPPP: Años acumulados de vida productiva potencial perdidos; DE: desviación estándar; CV: coeficiente de variación.

amputados por accidente laboral fue 41 a $60 \%$ de invalidez, con 42 casos, seguido del de $21-40 \%$, con 32 casos. Y el 68,5\% de la población presentó un porcentaje que oscilaba entre 21 y $60 \%$ de invalidez. De los pacientes que sufrieron la amputación por accidente laboral, 63,9\% no podrá realizar su labor habitual, 25\% lo hará con gran dificultad y 11,1\%, con dificultad leve. Así mismo, se encontró que de los pacientes amputados por accidente de trabajo, 75 (69,4\%) no estaban afiliados a algún tipo de seguro, pertenecían a EsSalud 29 (26,9\%), a sanidad 2 (1,9\%), a seguro privado $1(0,9 \%)$ y con seguro escolar $1(0,9 \%)$.

\section{DISCUSIÓN}

La determinación de los años acumulados de vida productiva potencial perdidos constituye un indicador importante para complementar la evaluación de las pérdidas ocasionadas por accidentes de trabajo.

La etiología traumática fue la más frecuente, seguido de la vascular del total de las amputaciones, durante el periodo de estudio. Esto es diferente a las referencias anteriores, que señalan la vascular en primer lugar ${ }^{(16)}$. Puede deberse al elevado número de accidentes de tránsito y propiamente a los accidentes laborales, que constituyeron 8,4\% del total de los pacientes amputados, lo que reafirma que las medidas de prevención son insuficientes.

En los 108 pacientes amputados por accidente laboral, se encontró una pérdida de 1 568,5 años acumulados de vida productiva potencial perdidos, con un promedio de 14,5 años por cada caso, con una desviación estándar de 8,5. La cantidad de AAVPPP disminuyó en los cinco años de estudio, encontrándose un menor número de casos en los últimos 3 años. El promedio bajó ligeramente, e incluso aumentó en el 2006, estando siempre por encima de 11 AAVPPP por cada trabajador afectado. El trabajo precedente al estudio tenía como promedio 3,2 años por caso, menor a nuestro estudio, pero hay que mencionar que abarcó todo tipo de impedimento y no solo amputaciones que originaran un mayor grado de invalidez ${ }^{(13)}$. García, Gadea y López en su estudio estimaron como promedio 2,9 años potenciales de vida laboral perdidos; pero, es de conocimiento que en este indicador solo considera las víctimas mortales ${ }^{(12)}$. Isidro, con el mismo indicador encontró 11,9 como promedio, pero fue en trabajadores mineros, en quienes hay mayor grado de lesión ${ }^{(11)}$. Velásquez señala que los años de vida saludables perdidos en el 2004 fue 183,4 por cada mil habitantes, pero es un indicador que suma los años de vida perdidos por muerte prematura y los años perdidos por discapacidad, y fue aplicado a la población general afectada por enfermedades y lesiones, sin diferenciar a la población laboral específicamente $^{(17)}$.

El nivel de amputación más frecuente fue debajo de la rodilla, seguido por dedos de la mano; el primero produce la mayor cantidad de años acumulados de vida productiva potencial perdidos, en el total de los pacientes, seguido por el debajo del codo.

La población que es afectada por un accidente laboral, en su mayoría es joven; se encontró un mayor porcentaje -40,7\%en el rango de 21 a 30 años. Similar a los reportes de los accidentes registrados en la DISA V, 57\% estuvo constituido por edades de 16 a 30 años ${ }^{(18)}$. Así mismo, $98,2 \%$ fue del sexo masculino, lo que indicaría que las actividades de mayor riesgo para accidentes de trabajo todavía las ejecuta el hombre. En el estudio en trabajadores de petróleos mexicanos, el sexo masculino representó 96,6\% ${ }^{(13)}$. Y en el de García, Gadea y López, 87\% fueron hombres, confirmando el alto número de accidentes de trabajo en este género ${ }^{(12)}$.

Las industrias manufactureras representó la actividad económica ${ }^{(19)}$ que presentó el mayor porcentaje de pacientes amputados por accidente laboral, seguido de transporte, almacenamiento y comunicaciones. También, los años acumula- 
dos de vida productiva potencial perdidos se encontraban en la misma distribución, pero en promedios de AAVPPP por caso. Correspondió en primer lugar a administración y defensa, seguido de explotación de minas y canteras; es decir, el daño fue mayor por persona. Esto se debería a que hay actividades con mayor frecuencia de accidentes, pero la gravedad de la lesión puede ser menor en comparación a otras actividades económicas, donde puede haber un mayor daño. Se debe diferenciar entre frecuencia de los accidentes de trabajo y la gravedad de la lesión que se produce según la actividad económica a que se dedica el trabajador. Informes del Ministerio de Salud ubican a los servicios médicos en primer lugar, con 48\%, seguido del de manufactura, con $25 \%$; pero, son registros de centros de salud de la DISA V, principalmente del personal de salud ${ }^{(18)}$.

La forma de accidente de mayor frecuencia fue el aprisionamiento o atrapamiento, seguido por el de choque de vehículos; la primera es característica en producir una amputación y la segunda, se debió al alto porcentaje de accidentes de tránsito. La exposición a fluidos biológicos $17 \%$, choques y golpes contra objetos $16 \%$ constituyen la referencia para accidentes laborales, en centros de salud principalmente ${ }^{(18)}$.

La amputación es la pérdida de un segmento que no se va recuperar, por lo cual es una invalidez permanente que se puede expresar en porcentaje. En el presente estudio, 38,9\% presentó un grado de invalidez de 41 a $60 \%$ y $68,5 \%$ tuvo invalidez que osciló entre 21 y $60 \%$, que generaron un porcentaje de invalidez importante. Así mismo, el desempeño de la labor habitual u ocupación que realizaba quedó anulado o muy disminuido, pues más de la mitad de la población estudiada no va a poder realizarla y la cuarta parte lo hará con gran dificultad. En muchos casos, dependiendo del daño, probablemente tendrá imposibilidad para trabajar, generando una carga familiar y costo para el país. Estos hallazgos no registran antecedentes y son aportes para futuras investigaciones.
Destaca en este estudio que $69,4 \%$ de los pacientes amputados por accidente de trabajo no contaba con un seguro, es decir pertenecerían al sector informal, que es el menos protegido; no cuentan con registros de trabajadores y menos cuando ocurre un accidente laboral, y con pobre información sobre salud ocupacional. Los hospitales públicos se convierten en un lugar estratégico para obtener información sobre este sector.

Es necesario desarrollar y mejorar las medidas preventivas contra accidentes de trabajo, en actividades económicas como la manufactura y el transporte, así como en actividades en que la gravedad del daño sea alta, que eviten la invalidez permanente y por consiguiente los años acumulados de vida productiva potencial perdidos. En estas acciones se debe involucrar a los empleadores, orientación sobre riesgos a los trabajadores jóvenes en los centros laborales, evaluar los conocimientos previos sobre seguridad y capacitarlos antes de empezar su labor.

Se debe considerar el indicador de años acumulados de vida productiva potencial perdidos para el análisis de los accidentes laborales; cuantifica la perdida de los años de vida productiva y es de fácil aplicación, lo que posibilitaría también una pensión más justa por invalidez.

\section{REFERENCIAS BIBLIOGRÁFICAS}

1. Normas Técnicas del Seguro Complementario de Trabajo de riesgo, Decreto Supremo No 003-98SA. El Peruano, No 6502, (14-04-1998).

2. Ministerio de Salud, DIGESA, OPS. Manual de Salud Ocupacional. Lima: Ministerio de Salud; 2005;30:73.

3. Ramírez C. Manual de Seguridad Industrial. 2a. ed. México: Limusa; 2006: 41.

4. Superintendencia de Administradoras Privadas de Fondos de Pensiones. Manual de Evaluación y Calificación del Grado de Invalidez. Perú: Dialogo S. A.; 1995.

5. Xhardez Y. Vademécum de Kinesioterapia y de Reeducación Funcional. Reimpresión. Argentina: El Ateneo; 1985:640.

6. Krusen F, Lehmann J. Krusen: Medicina Física y Rehabilitación. 4a ed. España: Médica Panamericana; 2000.
7. González MA, Cohí O, Salinas F. Amputación de extremidad inferior y discapacidad: Prótesis y rehabilitación. España: Elsevier; 2005:2.

8. Dirección de Salud V Lima Ciudad. Análisis de la Situación de Salud DISA V Lima Ciudad 2005. Perú: Ministerio de salud; 2005.

9. Chinchilla R. Salud y seguridad en el trabajo. Costa Rica: EUNED; 2002:95-7.

10. Lamata F. Manual de administración y gestión sanitaria. España: Díaz de Santos; 1998:646.

11. Isidro J. Factores determinantes del impacto económico de los años de vida productiva potencialmente perdidos (AVPPP) y la magnitud de los años de vida potenciales perdidos (AVPP) ocasionados por accidentes de trabajo mortales en la minería peruana 1994-1999 [tesis de maestría]. Perú: Unidad de Postgrado de Medicina, Universidad Nacional Mayor de San Marcos; 2003.

12. García A, Gadea R, López V. Estimación de la mortalidad atribuible a enfermedades laborales en España, 2004. Rev Esp Salud Publica. 2007;81(3):261-70.

13. Güemes JC, Mouriño RR, Páez J, Muñoz RL. Años acumulados de vida productiva potencial perdidos por accidentes de trabajo en Petróleos Mexicanos. Salud Publica Mex. 1996;38(2):110-7.

14. Instituto Nacional de Rehabilitación [sede Web]. Callao: inr.gob.pe; 2004 [acceso 20 junio de 2009]. Disponible en: http://www.inr.gob.pe .

15. Modificación del Régimen de Prestaciones de Salud, el Sistema Nacional de Pensiones, el Sistema Privado de Fondos de Pensiones y la estructura de Contribuciones al Fonavi, Ley $N^{\circ}$ 26504. El Peruano, № 5487, (18-07-1995).

16. Serra G. Fisioterapia en traumatología, ortopedia y reumatología. 2a ed. España: Elsevier; 2006:191.

17. Velásquez A. La carga de enfermedad y lesiones en el Perú y las prioridades del plan esencial de aseguramiento universal. Rev Peru Med Exp. 2009;26(2):222-31.

18. Dirección de Salud V Lima Ciudad. Análisis de la Situación de Salud DISA V Lima Ciudad 2006. Perú: Ministerio de salud; 2006:179-83.

19. Naciones Unidas. Clasificación Industrial Internacional Uniforme de todas las Actividades económicas (CIIU). Nueva York: United Nations Publications; 2005:49-217.

Trabajo recibido el 12 de octubre de 2010 y aceptado para publicación el 28 de octubre de 2010.

Correspondencia:

Calle Ícaro 105 Urb. La Campiña, Chorrillos

Teléfonos: 4672103 - 999251213

Correo electrónico: teocamacho20@hotmail.com 